July 2021

\title{
Additional Baryons and Mesons
}

\author{
Paul H. Frampton* \\ Dipartimento di Matematica e Fisica "Ennio De Giorgi", \\ Università del Salento and INFN-Lecce, \\ Via Arnesano, 73100 Lecce, Italy
}

\begin{abstract}
In a particle theory model whose most readily discovered new particle is the $\sim 1 \mathrm{TeV}$ bilepton resonance in same-sign leptons, currently being sought at CERN's LHC, there exist three quarks $\mathcal{D}, \mathcal{S}, \mathcal{T}$ which will be bound by QCD into baryons and mesons. We consider the decays of these additional baryons and mesons whose detailed experimental study will be beyond the reach of the $14 \mathrm{TeV}$ CERN collider and accessible only at an $\mathrm{O}(100 \mathrm{TeV})$ collider.
\end{abstract}

*paul.h.frampton@gmail.com 
An important question in high-energy physics is what will be the first new particle to be discovered beyond the standard model. In the Letter, we discuss a possible answer to this question and how our answer could impact on particle phenomenology beyond the TeV energy scale.

A particle theory model which explains the occurrence of three quark-lepton families in the standard model [1-3] is the bilepton model. For the minimal 331-model with no additional leptons, as proposed in [4], this involves, inter alia, doubly-charged gauge bosons $Y^{ \pm \pm}$with lepton number $|L|=2$ which decay into same-sign leptons and are being sought at the LHC. Some of the relevant LHC phenomenology is discussed in $[5,6]$. A refined mass estimate derived in [7] is $M\left(Y^{ \pm \pm}\right)=(1.29 \pm 0.06) \mathrm{TeV}$ where faute de mieux it was assumed that the symmetry breaking of $S U(3)_{L}$ is closely similar to that of $S U(2)_{L}$. It will be pleasing if the physical mass is consistent with this.

Because the quarks are in triplets and anti-triplets of $S U(3)_{L}$, rather than only in doublets of $S U(2)_{L}$ as in the standard model, there is necessarily an additional quark in each family. In the first and second families they are the $\mathcal{D}$ and $\mathcal{S}$ respectively, both with charge $Q=-4 / 3$ and lepton number $L=+2$. In the third family is the $\mathcal{T}$ with charge $Q=+5 / 3$ and lepton number $L=-2$. All the three $\mathrm{TeV}$ scale quarks are colour triplets with spin- $\frac{1}{2}$ and baryon number $B=\frac{1}{3}$. Their masses are yet to be measured but may be expected to be below the ceiling of $4.1 \mathrm{TeV}$ which is the upper limit for symmetry breaking of $S U(3)_{L}$ and probably above $1 T e V$. By analogy with the known quarks, one might expect $M(\mathcal{T})>M(\mathcal{S})>M(\mathcal{D})$, although without experimental data this is conjecture.

The heavy quarks and antiquarks will be bound to light quarks and antiquarks, and to each other, to form an interesting spectroscopy of mesons and baryons. Let us first display, in Tables 1, 2 the TeV mesons, then in Tables $3,4,5$ the $\mathrm{TeV}$ baryons. The charge conjugate states are equally expected, and will reverse the signs of $Q$ and $L$. 
Table 1: TeV mesons $\mathcal{Q} \bar{q}$

\begin{tabular}{||c|c||c|c||}
\hline \hline $\mathcal{Q}$ & $\bar{q}$ & $\mathrm{Q}$ & $\mathrm{L}$ \\
\hline \hline & & & \\
$\mathcal{D} / \mathcal{S}$ & $\bar{u}$ etc. & -2 & +2 \\
$\mathcal{D} / \mathcal{S}$ & $\bar{d}$ etc. & -1 & +2 \\
$\mathcal{T}$ & $\bar{u}$ etc. & +1 & -2 \\
$\mathcal{T}$ & $\bar{d}$ etc. & +2 & -2 \\
& & & \\
\hline \hline
\end{tabular}

Table 2: $\mathrm{TeV}$ mesons $\mathcal{Q} \overline{\mathcal{Q}}$

\begin{tabular}{||c|c||c|c||}
\hline \hline $\mathcal{Q}$ & $\overline{\mathcal{Q}}$ & $\mathrm{Q}$ & $\mathrm{L}$ \\
\hline $\mathcal{D} / \mathcal{S}$ & $\overline{\mathcal{D}} / \overline{\mathcal{S}}$ & 0 & 0 \\
$\mathcal{D} / \mathcal{S}$ & $\overline{\mathcal{T}}$ & -3 & +4 \\
$\mathcal{T}$ & $\bar{T}$ & 0 & 0 \\
\hline \hline
\end{tabular}

Although the $\mathcal{Q}$ masses are unknown, it may be reasonable first to make a preliminary discussion of these states by assuming that

$$
M(\mathcal{T})>M(\mathcal{S})+2 M_{t}>M(\mathcal{D})+4 M_{t}
$$

where $M_{t}$ is the top quark mass so that the lightest of the TeV baryons and mesons are those containing just one $\mathcal{D}$ quark or one $\overline{\mathcal{D}}$ antiquark. The next lightest are the $\mathrm{TeV}$ baryons and mesons containing just one $\mathcal{S}$ quark or one $\overline{\mathcal{S}}$ antiquark. 
Table 3: TeV baryons $\mathcal{Q} q q$

\begin{tabular}{||c|c||c|c||}
\hline \hline $\mathcal{Q}$ & qq & $\mathrm{Q}$ & $\mathrm{L}$ \\
\hline $\mathcal{D} / \mathcal{S}$ & dd etc. & -2 & +2 \\
$\mathcal{D} / \mathcal{S}$ & ud etc. & -1 & +2 \\
$\mathcal{D} / \mathcal{S}$ & uu etc. & 0 & +2 \\
$\mathcal{T}$ & dd etc. & +1 & -2 \\
$\mathcal{T}$ & ud etc. & +2 & -2 \\
$\mathcal{T}$ & uu etc, & +3 & -2 \\
\hline \hline
\end{tabular}

Table 4: TeV baryons $\mathcal{Q} \mathcal{Q} q$

\begin{tabular}{||c|c||c|c||}
\hline \hline $\mathcal{Q Q}$ & $q$ & $\mathrm{Q}$ & $\mathrm{L}$ \\
\hline$(\mathcal{D} / \mathcal{S})(\mathcal{D} / \mathcal{S})$ & d etc. & -3 & +4 \\
$(\mathcal{D} / \mathcal{S})(\mathcal{D} / \mathcal{S})$ & u etc. & -2 & +4 \\
$(\mathcal{D} / \mathcal{S}) \mathcal{T}$ & d etc. & 0 & 0 \\
$(\mathcal{D} / \mathcal{S}) \mathcal{T}$ & u etc. & +1 & 0 \\
$\mathcal{T} \mathcal{T}$ & d etc. & +3 & -4 \\
$\mathcal{T} \mathcal{T}$ & u etc. & +4 & -4 \\
\hline
\end{tabular}

We begin by discussing the decay modes of the $\mathcal{D} \bar{q}$ mesons in Table 1 , focusing on final states from the first family. The decays of $\mathcal{D}$ include, taking care of $L$ conservation,

$$
\begin{aligned}
\mathcal{D} & \rightarrow d+Y^{-} \\
& \rightarrow d+\left(e^{-}+\nu_{e}\right) \\
& \rightarrow d+\left(\mu^{-}+\nu_{\mu}\right) \\
& \rightarrow d+\left(\tau^{-}+\nu_{\tau}\right)
\end{aligned}
$$


Table 5: TeV baryons $\mathcal{Q Q} \mathcal{Q}$

\begin{tabular}{||c||c|c||}
\hline \hline $\mathcal{Q Q} \mathcal{Q}$ & $\mathrm{Q}$ & $\mathrm{L}$ \\
\hline & & \\
$(\mathcal{D} / \mathcal{S})(\mathcal{D} / \mathcal{S})(\mathcal{D} / \mathcal{S})$ & -4 & +6 \\
$(\mathcal{D} / \mathcal{S})(\mathcal{D} / \mathcal{S}) \mathcal{T}$ & -1 & +2 \\
$(\mathcal{D} / \mathcal{S}) \mathcal{T} \mathcal{T}$ & +2 & -2 \\
$\mathcal{T} \mathcal{T} \mathcal{T}$ & +5 & -6 \\
& & \\
\hline \hline
\end{tabular}

which implies that decays of the $(\mathcal{D} \bar{u})$ meson include

$$
\begin{aligned}
(\mathcal{D} \bar{u}) & \rightarrow \pi^{-}+\left(e^{-}+\nu_{e}\right) \\
& \rightarrow \pi^{-}+\left(\mu^{-}+\nu_{\mu}\right) \\
& \rightarrow \pi^{-}+\left(\tau^{-}+\nu_{\tau}\right)
\end{aligned}
$$

and variants thereof where $\pi^{-}$is replaced by any other non-strange negatively charged meson. The $d$ in Eq.(2) can be replaced by $s$ or $b$ which subsequently decay.

An alternative to Eq.(2) is

$$
\begin{aligned}
\mathcal{D} & \rightarrow u+Y^{--} \\
& \rightarrow u+\left(e^{-}+e^{-}\right) \\
& \rightarrow u+\left(\mu^{-}+\mu^{-}\right) \\
& \rightarrow u+\left(\tau^{-}+\tau^{-}\right)
\end{aligned}
$$

which implies additional decay modes of the $(\mathcal{D} \bar{u})$ meson which include

$$
\begin{aligned}
(\mathcal{D} \bar{u}) & \rightarrow \pi^{0}+\left(e^{-}+e^{-}\right) \\
& \rightarrow \pi^{0}+\left(\mu^{-}+\mu^{-}\right) \\
& \rightarrow \pi^{0}+\left(\tau^{-}+\tau^{-}\right)
\end{aligned}
$$

and variants obtained by flavour replacements. Eqs.(3) and (5), and their generalisations to other flavours, suffice to illustrate the richness of $(\mathcal{D} \bar{u})$ decays. 
Turning to the meson $\mathcal{D} \bar{d}$, we can use Eq.(2) to identify amongst its possible decays

$$
\begin{aligned}
(\mathcal{D} \bar{d}) & \rightarrow \pi^{0}+\left(e^{-}+\nu_{e}\right) \\
& \rightarrow \pi^{0}+\left(\mu^{-}+\nu_{\mu}\right) \\
& \rightarrow \pi^{0}+\left(\tau^{-}+\nu_{\tau}\right)
\end{aligned}
$$

and variants thereof where $\pi^{0}$ is replaced by any other non-strange neutral meson. When $u$ in Eq.(2) is replaced by $c$ or $t$ which subsequently decay, we arrive at many other decay channels additional to Eq.(6).

Employing instead the $\mathcal{D}$ decays in Eq.(4) implies additional decay modes of $(\mathcal{D} \bar{d})$ meson that include

$$
\begin{aligned}
(\mathcal{D} \bar{d}) & \rightarrow \pi^{+}+\left(e^{-}+e^{-}\right) \\
& \rightarrow \pi^{+}+\left(\mu^{-}+\mu^{-}\right) \\
& \rightarrow \pi^{+}+\left(\tau^{-}+\tau^{-}\right)
\end{aligned}
$$

and variants obtained by flavour replacement. Eqs.(6) and (7), merely illustrate a few of the simplest $(\mathcal{D} \bar{d})$ decays. There are many more.

Next we consider the lightest $\mathrm{TeV}$ baryons in Table 3 with $\mathcal{Q}=\mathcal{D}$. Using the $\mathcal{D}$ decays from Eq. (2) we find for $(\mathcal{D} u u)$ decay

$$
(\mathcal{D} u u) \rightarrow p+\left(l_{i}^{-}+\nu_{i}\right) .
$$

together with flavour rearrangements. Here, as in subsequent equations, $i=e, \mu, \tau$.

Alternatively, the $\mathcal{D}$ decays from Eq.(4) lead to

$$
\begin{aligned}
(\mathcal{D} u u) & \rightarrow N^{*++}+Y^{--} . \\
& \rightarrow p+\pi^{+}+\left(l_{i}^{-}+l_{i}^{-}\right) . .
\end{aligned}
$$

Looking at the $\mathrm{TeV}$ baryon $(\mathcal{D} u d)$ the respective sets of decays corresponding to Eq.(2) are

$$
(\mathcal{D} u d) \rightarrow n+\left(l_{i}^{-}+\nu_{i}\right)
$$


where only the simplest light baryon is exhibited.

Corresponding to $\mathcal{D}$ decays in Eq.(4) there are also

$$
(\mathcal{D} u d) \rightarrow p+\left(l_{i}^{-}+l_{i}^{-}\right)
$$

in the simplest cases.

Finally, of the $(\mathcal{D} q q) \mathrm{TeV}$ baryons, we write out the decays for $(\mathcal{D} d d)$, first for the $\mathcal{D}$ decays in Eq. $(2)$

$$
\begin{aligned}
(\mathcal{D} d d) & \rightarrow N^{*-}+Y^{-} \\
& \rightarrow n+\pi^{-}+\left(l_{i}^{-}+\nu_{i}\right) .
\end{aligned}
$$

within flavour variations.

With the Eq.(4) decays of $\mathcal{D}$ there are finally decays of the sort

$$
(\mathcal{D} d d) \rightarrow n+\left(l_{i}^{-}+l_{i}^{-}\right)
$$

again with more possibilities by choosing alternative flavours.

We now replace the TeV quark $\mathcal{D}$ by the next heavier $\mathrm{TeV}$ quark $\mathcal{S}$ and repeat our study of decays whereupon we shall encounter the first example of decay not only to the known quarks but also to a TeV quark. 
The TeV quark $\mathcal{S}$ has possible decay channels

$$
\begin{aligned}
\mathcal{S} & \rightarrow d+Y^{-} \\
& \rightarrow d+\left(e^{-}+\nu_{e}\right) \\
& \rightarrow d+\left(\mu^{-}+\nu_{\mu}\right) \\
& \rightarrow d+\left(\tau^{-}+\nu_{\tau}\right) \\
& \rightarrow \mathcal{D}+Z^{\prime} \\
& \rightarrow d+\left(e^{-}+\nu_{e}\right)+\left(e^{+}+e^{-}\right) \\
& \rightarrow d+\left(e^{-}+\nu_{e}\right)+\left(\mu^{+}+\mu^{-}\right) \\
& \rightarrow d+\left(e^{-}+\nu_{e}\right)+\left(\tau^{+}+\tau^{-}\right) \\
& \rightarrow d+\left(\mu^{-}+\nu_{\mu}\right)+\left(e^{+}+e^{-}\right) \\
& \rightarrow d+\left(\mu^{-}+\nu_{\mu}\right)+\left(\mu^{+}+\mu^{-}\right) \\
& \rightarrow d+\left(\mu^{-}+\nu_{\mu}\right)+\left(\tau^{+}+\tau^{-}\right) \\
& \rightarrow d+\left(\tau^{-}+\nu_{\tau}\right)+\left(e^{+}+e^{-}\right) \\
& \rightarrow d+\left(\tau^{-}+\nu_{\tau}\right)+\left(\mu^{+}+\mu^{-}\right) \\
& \rightarrow d+\left(\tau^{-}+\nu_{\tau}\right)+\left(\tau^{+}+\tau^{-}\right)
\end{aligned}
$$

where we note the opening up of channels due to $\mathcal{S} \rightarrow \mathcal{D}$ decay.

With Eq.(14) in mind, the decays of the TeV meson $(\mathcal{S} \bar{u})$ include

$$
\begin{aligned}
(\mathcal{S} \bar{u}) & \rightarrow \pi^{-}+\left(l_{i}^{-}+\nu_{i}\right) \\
& \rightarrow \pi^{-}+\left(l_{i}^{-}+\nu_{i}\right)+\left(l_{j}^{+}+l_{j}^{-}\right)
\end{aligned}
$$

where the second line involves a $\mathcal{D}$ intermediary.

An alternative to Eq.(14) is

$$
\begin{aligned}
\mathcal{S} & \rightarrow u+Y^{--} \\
& \rightarrow u+\left(e^{-}+e^{-}\right) \\
& \rightarrow u+\left(\mu^{-}+\mu^{-}\right) \\
& \rightarrow u+\left(\tau^{-}+\tau^{-}\right)
\end{aligned}
$$

which implies additional decay modes of $(\mathcal{S} \bar{u})$

$$
(\mathcal{S} \bar{u}) \rightarrow \pi^{0}+\left(l_{i}^{-}+l_{i}^{-}\right)
$$


and variants which replace $\pi^{0}$ by another neutral non-strange meson. Eqs.(15) and (17), illustrate sufficiently $(\mathcal{S} \bar{u})$ decays.

Turning to the meson $(\mathcal{S} \bar{d})$, we can use Eq.(14) to identify its possible decays

$$
(\mathcal{S} \bar{d}) \rightarrow \pi^{0}+\left(l_{i}^{-}+\nu_{i}\right)
$$

When $u$ in Eq.(14) is replaced by $c$ or $t$ which subsequently decay, we arrive at many other decay channels additional to Eq.(18).

Employing instead the $\mathcal{S}$ decays in Eq.(16) implies additional decay modes of $(\mathcal{S} \bar{d})$ that include

$$
(\mathcal{S} \bar{d}) \rightarrow \pi^{+}+\left(l_{i}^{-}+l_{i}^{-}\right)
$$

and variants obtained by flavour replacement. Eqs.(18) and (19), illustrate only a few of the simplest $(\mathcal{S} \bar{d})$ decays. There are many more.

Next we consider the lightest $\mathrm{TeV}$ baryons in Table 3 with one $\mathcal{Q}=\mathcal{S}$. Using the $\mathcal{S}$ decays from Eq.(14) we find for $(\mathcal{S} u u)$ decay

$$
(\mathcal{S} u u) \rightarrow p+\left(l_{i}^{-}+\nu_{i}\right) .
$$

together with flavour rearrangements.

Alternatively, the $\mathcal{S}$ decays from Eq.(16) lead to

$$
\begin{aligned}
(\mathcal{S} u u) & \rightarrow N^{*++}+\left(l_{i}^{-}+l_{i}^{-}\right) . . \\
& \rightarrow p+\pi^{+}+\left(l_{i}^{-}+l_{i}^{-}\right) .
\end{aligned}
$$

Looking at the $\mathrm{TeV}$ baryon $(\mathcal{S} u d)$ the respective sets of decays corresponding to Eq.(14) are

$$
(\mathcal{S} u d) \rightarrow n+\left(l_{i}^{-}+\nu_{i}\right)
$$

where only the simplest version is exhibited.

Corresponding to the $\mathcal{S}$ decays in Eq.(16) there are the decays

$$
(\mathcal{S} u d) \rightarrow p+\left(l_{i}^{-}+l_{i}^{-}\right)
$$


For baryon $(\mathcal{S} d d)$, firstly from the $\mathcal{S}$ decays in Eq.(14) we have

$$
\begin{aligned}
(\mathcal{S} d d) & \rightarrow N^{*-}+Y^{-} \\
& \rightarrow n+\pi^{-}+\left(l_{i}^{-}+\nu_{i}\right) .
\end{aligned}
$$

within flavour variations.

Secondly, from the Eq.(16) decays of $\mathcal{S}$ there are baryon decays of the type

$$
(\mathcal{S} d d) \rightarrow n+\left(l_{i}^{-}+l_{i}^{-}\right)
$$

with more possibilities by choosing alternative flavours.

We could continue further to study decays of all the baryons and mesons in our Tables. However, it seems premature to do so until we know from experimental data the masses and mixings of $\mathcal{D}, \mathcal{S}, \mathcal{T}$. We remark only that the type of lepton cascade which we have deliberately exhibited explicitly in Eq.(14) becomes a more prevalent possibility as the lepton number of the decaying hadron increases.

We may expect, by analogy with the top quark, that the mass of the $\mathcal{T}$ quark, although probably below $4.1 \mathrm{TeV}$ for the symmetry-breaking reason discussed ut supra might be not much below. For example it might exceed 3 $\mathrm{TeV}$ whereupon the mass of a $(\mathcal{T} \mathcal{T} \mathcal{T})$ baryon could exceed $9 \mathrm{TeV}$. Since this baryon has high lepton number, it is pair produced and such pair production is beyond the reach of the $14 \mathrm{TeV}$ LHC. Its study requires a $100 \mathrm{TeV}$ collider of the type under discussion at CERN and in China. As a foretaste of the physics, one notable decay of the $(\mathcal{T} \mathcal{T} \mathcal{T})$ baryon is into $\left[p+4\left(e^{+}\right)+2\left(\bar{\nu}_{e}\right)\right]$.

At the time of writing, the particles exhibited in our Tables are conjectural. If the bilepton is established as the first new elementary particle discovered since $2012[8,9]$, the existence of all the additional baryons and mesons will become a sharp prediction.

\section{Acknowlegement}

We thank the University of Salento for affiliation and S.L.Glashow for supporting a bilepton search at CERN. 


\section{References}

[1] S.L. Glashow, Partial Symmetries of the Weak Interactions.

Nucl. Phys. 22, 579 (1961).

[2] S. Weinberg, A Model of Leptons.

Phys. Rev. Lett. 19, 1264 (1967).

[3] A. Salam,

Weak and Electromagnetic Interactions.

Contribution to the 8th Nobel Symposium, p 367 (1968).

[4] P.H. Frampton, Chiral Dilepton Model and the Flavor Question.

Phys. Rev. Lett. 69, 2889 (1992).

[5] G. Corcella, C. Coriano, A. Costantini and P.H. Frampton, Bilepton Signatures at the $\mathrm{LHC}$.

Phys.Lett. B773, 544 (2017).

arXiv:1707.01381 [hep-ph].

[6] G. Corcella, C. Coriano, A. Costantini and P.H. Frampton, Exploring Scalar and Vector Bileptons at the LHC in a 331 Model.

Phys.Lett. B785, 73 (2018).

arXiv:1806.04536 [hep-ph].

[7] C. Coriano and P.H. Frampton, Refined Mass Estimate for Bilepton Gauge Boson.

Mod. Phys. Lett. A (2021, in press).

arXiv:2011.02037 [hep-ph].

[8] G. Add, et al. (ATLAS Collaboration), Observation of a New Particle in the Search for the Standard Model Higgs Boson with the ATLAS Detector at CERN.

Phys. Lett. B716, 1 (2012).

arXiv: 1207 . 7214 [hep-ex] .

[9] S. Chatrchyan, et al. (CMS Collaboration).

Observation of a New Boson at a Mass 125 GeV with the CMS Experiment at the $L H C$.

Phys. Lett. B716, 30 (2012).

arXiv: 1207.7235 [hep-ex] . 